\title{
Photoacoustic Microscopy in Dental Medicine
}

\author{
Adrian Tudor Stan1, Laura Idorași1, Victor Florin Stan1, Alexandru Florin Rogobete², \\ Cosmin Sinescu¹, Meda Lavinia Negruțiu', Mihai Romînu¹ \\ 1 Faculty of Dental Medicine, "Victor Babeș” University of Medicine and Pharmacy, Timișoara, Romania \\ 2 Faculty of Medicine, "Victor Babeș" University of Medicine and Pharmacy, Timișoara, Romania
}

\section{CORRESPONDENCE}

\section{Adrian Tudor Stan}

P-ța Eftimie Murgu nr. 2

300041 Timișoara, Romania

Tel: +40 256204400

E-mail: stanadrian7@yahoo.com

\section{ARTICLE HISTORY}

Received: 3 March, 2017

Accepted: 9 March, 2017
Laura Idorași • P-ța Eftimie Murgu nr. 2, 300041 Timișoara, Romania, Tel: +40 256204400 Victor Florin Stan • P-ța Eftimie Murgu nr. 2, 300041 Timișoara, Romania, Tel: +40 256204400 Alexandru Florin Rogobete • P-ța Eftimie Murgu nr. 2 300041 Timișoara, Romania, Tel: +40 256204400 Cosmin Sinescu • P-ta Eftimie Murgu nr. 2, 300041 Timișoara, Romania, Tel: +40 256204400 Meda Lavinia Negrutiu • P-ta Eftimie Murgu nr. 2 , 300041 Timișoara, Romania, Tel: +40 256204400 Mihai Romînu • P-ța Eftimie Murgu nr. 2, 300041 Timișoara, Romania, Tel: +40 256204400

\begin{abstract}
Introduction: Photoacoustic microscopy, also known as optoacoustic imaging, is a comparatively new method of investigation in dental medicine, which uses a laser-generated ultrasound (short laser pulses) to achieve images for interpretation. Photoacoustic microscopy can be used in a broad spectrum, from detecting tooth decay at its earliest stages to dental anatomy analysis. Material and methods: The energy emitted by the photoacoustic pulse is moderately absorbed by the target and exchanged into heat, leading to a local transitory temperature upsurge. The tension propagates and grows as ultrasonic waves, distinguished by the ultrasonic transducers which are planted apart from the tissue. The photoacoustic microscope has a tunable dye laser which passes through a condensing lens, an objective and ultimately an ultrasonic transducer attached to an acoustic lens to capture and receive information about the scanned probe from a sample moved on the $X, Y$ dimensions. Results: The precise anatomy of layered concentric structures can be clearly observed in photoacoustic microscopy. The image value of the inner layer can be higher, indicating strong optical absorption, while the image value of the outer layer is lower, indicating weaker optical absorption. Meanwhile, the inner layer has the exact same size as the dentin structure and the outer layer has the exact same size as the enamel structure in this cross-section. Conclusions: The photoacoustic microscope (all-optical) comes out to be a future and promising tool for detecting early-stage caries and lesions on the surface of the teeth, where micro-leakage occurs at the interface of tooth restoration, and also the anatomy of dental tissues.
\end{abstract}

Keywords: biomedical imaging, photoacoustic microscopy, dentistry, human teeth, marginal adaptation

\section{INTRODUCTION}

Photoacoustic microscopy, also known as optoacoustic imaging, is a comparatively new method of investigation in dental medicine, which uses a laser-generated ultrasound (short laser pulses) to achieve images for interpretation. Photoacoustic microscopy can be used in a broad spectrum, from detecting tooth decay at its earliest stages to dental anatomy analysis. The feasibility of a photoacoustic microscopy system using a common, commercial ultrasound scanner in order to retrieve the acoustic data, is to be determined. It is a hybrid method, 
mixing the interaction between hard-matter and electromagnetic radiation, resulting in a very high contrast and very high resolution optical image. ${ }^{1,2}$

Photoacoustic imaging analyses are similar to other imaging techniques regarding rigorous depth data, submillimeter resolution and nano-molar sensitivity. With the latest advances in background cutback, as well as the manipulation of lasers with high repetition rates, it is very likely that photoacoustic tomography will find its place in the near future in both preclinical dental research and clinical use. The purpose of this paper is to discuss and analyze recent advances in photoacoustic imaging and to see the prospects of its future applications in dental medicine. ${ }^{1,3}$

Photoacoustic imaging can be classified into two types of systems: photoacoustic computed tomography (PACT) and photoacoustic microscopy (PAM). PACT operates by reconstructing algorithms to generate a set of images, while PAM uses direct point-by-point detection in addition to scanning over an object to provide a result. ${ }^{2,4}$

\section{MATERIALS AND METHODS, CURRENT SITUATION, CHALLENGES}

\subsection{Photoacoustic microscope}

Photoacoustic imagery (PI) is rooted in the foundations of the photoacoustic effect, which was primarily investigated by A.G. Bell in 1880. Commonly, the PI effect starts from a target point in the samples treated with a small laser pulse. The low-to-moderate energy radiated by the pulse is captured by the sample and exchanged into heat, leading to a confined transitory temperature upswing, followed by a tension and pressure rise due to thermo-elastic extension. The tension increasingly grows and spreads in the form of ultrasonic waves, known as photoacoustic imaging waves. ${ }^{1,5}$

Virtually, the photoacoustic method measures the change of electromagnetic energy emitted by the source into acoustic pressure waves. In biomedical PI, the sample is irradiated with a nanosecond pulsed laser, generating an ultrasound wave due to the optical absorption and thermal expansion of the scanned sample. By distinguishing the pressure waves applied with the ultrasound transducer, the image will be assembled with the primary contrast in relation with the optical absorption of the sample or tissue. This instrument, through which a photoacoustic image is acquired, provides certain advantages compared to other ex vivo imaging techniques. ${ }^{1,5,6}$

The contrast distinction instrument in photoacoustic imagery is based on the diversity in the optical absorp- tion attributes of the tissue or sample components. Photoacoustic imaging is suitable for imaging frameworks with great optical coefficient, such as blood vessels in the pulp of the teeth, but with its dual style, it can also be used for hard tissue surfaces, such as enamel or dentine. On the other hand, photoacoustic imaging can be obtained by utilizing longer wavelengths in the near-infrared specter, where the sample absorption is minimal. Light is able to pass through a couple of centimeters into biological samples or tissues, and the working wavelengths are in the near-infrared specter, at the same time remaining beneath the safe laser exposure limits of the tissue. The photoacoustic mechanism penetrates more profoundly into tissues than conventional optical imaging techniques that use ballistic or quasi-ballistic photons such as optical coherence tomography. Photoacoustic microscopy does not depend on the detection of photons alternatively, weak acoustic waves are scattered and detected as a feedback to laser irradiation. Moreover, a photoacoustic imaging mechanism can be easily mixed with an ultrasound technique, considering that both systems can have almost the same detector and electronic parts. The dominant contrast in ultrasonic imaging comes from the mechanical attributes of the sample or tissue, which frequently expresses anatomical information. Therefore, combined photoacoustic and ultrasound techniques can contribute to both anatomical and, partly, functional information. ${ }^{1,3,7}$

The photoacoustic microscope has a tunable dye laser, which passes through a condensing lens, an objective, and ultimately an ultrasonic transducer attached to an acoustic lens to capture and receive information about the scanned probe from a sample moved on the $\mathrm{X}, \mathrm{Y}$ dimensions.

\subsection{Current situation}

Tooth solid tissue (enamel, dentine and cementum) lesions, along with caries, cracked teeth etc., are among the most frequent diseases that affect mankind. Tooth lesions decrease the quality of life of patients, and it is still a challenge to noninvasively detect dental lesions in their early stages, when they cannot be viewed with the naked eye. ${ }^{8}$

Photoacoustic imaging is an emerging hybrid technology that combines the high spatial resolution of ultrasound waves in profound tissues with rich optical contrast. Dualcontrast photoacoustic microscopy or tomography can be used to detect dental caries and lesions in their early stages. One contrast, named B-mode, is linked to optical absorption and provides sharp images of the morphological aspects of the teeth. Another contrast, named S-mode, is associated with the microstructural and mechanical 
characteristics of dental hard tissues, being able to detect changes in tissue characteristics caused by premature tooth lesions. Furthermore, the S-mode imaging parameter is independent of the device and is able to quantify tissue properties. This system could improve diagnosis accuracy, specificity and sensitivity in case of dental lesions. ${ }^{8,9}$

Moreover, with the help of photoacoustic waves, hemoglobin solutions in dental roots can be identified using an ultrasonic soft probe situated on a composite transducer on the surface of the tooth. The high-frequency ultrasonic waves identify the hemoglobin in the pulp cavity due to its high heat transfer and absorption coefficient. ${ }^{9}$

\subsection{Challenges}

As far as laser safety is concerned, during photoacoustic imaging the laser light can make the skin become red and irradiated. For safety reasons, it is recommended that the laser radiation be kept beneath the maximum permissible exposure (MPE), which is the maximum amount of laser radiation to which a human tissue can be exposed to, without the occurrence of hazardous effects or biological modifications. The MPE of human skin to a pulsed laser, determined by the American National Standards Institute, is $20-100 \mathrm{~mJ} / \mathrm{cm}^{2}$, with wavelengths in the $400-1300 \mathrm{~nm}$ range, reaching even $1500 \mathrm{~nm}$. Higher laser radiation produces higher noise in photoacoustic imaging, and exceeding the MPE increases the temperature of the irradiated tissue and has the potential to create pain or even burn the patient's skin. ${ }^{1-4}$

Photoacoustic imaging with external variation agents is a rapidly developing field due to the benefits described above. Nonetheless, the long-term toxicity and the accumulating nanoparticles continue to pose a concern. The toxicity and the risk of accumulation are variable, based on the contrast between sizes, spatial geometries and surface morphology of nanoparticles. For the nanoparticles to be used in a clinical setting, toxicity studies are needed for each type of nanoparticle. Several studies aimed to assess long-term safety threats in vivo, as nanoparticles larger than a specific diameter (approximately 5-6 nm) cannot have a proper renal clearance and will deposit in important organs such as the liver and the spleen. Biodegradable bundles of nanoparticles were newly introduced as a promising means to address the long clearance time and long-term adverse effects of metallic nanoparticles. ${ }^{1-3}$

The temporal resolution of current photoacoustic imaging methods is an important and hindering problem. Photoacoustic mechanisms used with optical parametric oscillator (OPO) lasers are mostly working at low frame rates due to the low pulse repetitions of the laser pulse. Recently, attempts have been made to raise the temporal resolution of photoacoustic imaging with the scope of developing real-time photoacoustic imaging mechanisms. ${ }^{1,7}$

The expenditure and portability can be summated within the major cost associated with a photoacoustic imaging system and it is due to the need for costly Q-switched lasers. The use of low-cost compact laser diodes that have wide emission wavelength availability rather than conventional Q-switched pulsed mechanisms, frees the prospect of creating portable and miniaturized imaging instrumentation applicable for clinical uses. ${ }^{1,3}$

\section{DISCUSSIONS}

From small tissues to the whole body, from microvasculature to internal viscera, from morphology to function, photoacoustic microscopy and tomography are poised to have an increasingly relevant role in basic physiological research in dental medicine, especially in pre-clinical studies. It is a very rapidly growing field, one which has newly emerged in dental medicine in the last couple of years.

Assimilation of the most advanced technologies will eventually lead to the commercialization of photoacoustic microscopy and tomography for clinical activity. For microscopy, a rapid scanning system and an increased laser replication rate with an extensive series of wavelengths are needed for real-time functional imaging with no alteration of the spatial resolution. Moreover, an improved optical and acoustic focal point technique is necessary to sustain the best resolution in depth. In case of tomography, novel mechanisms for assembling ultrasound designs will help boost imaging sensitivity, specificity and enhance the spatial resolution. Additional consistent beam development above the tissue surface is essential for whole-field illumination. Comparably, the retrieval of data is performed real-time and is relevant for the additional improvement of imaging speed. 5,6

Photoacoustic tomography can be safely used for clinical applications as it delivers an optical fluency that falls within the safety standards of the American National Standards Institute. Yet, mechanical improvement is needed to facilitate an adequate clinical translation. ${ }^{1,7}$

Imagery that provides complementary information for more precise diagnosis is always favorable. Photoacoustic tomography can be combined with confocal microscopy, optical coherence tomography, ultrasound imaging, or magnetic resonance imaging in order to provide beneficial anatomical, functional and molecular information. Current photoacoustic microscopy studies are putting an em- 
phasis on miniaturizing the mechanism and improving our understanding of the wave algorithms. ${ }^{10,11}$ In summary of the discussions, photoacoustic microscopy and tomography are excellent companions of other biomedical imagistic techniques by offering particular optical absorption variation and contrast with very high scalable spatial resolution, penetration depth and speed..$^{1,3,12}$

\section{CONCLUSIONS}

The photoacoustic microscope (all-optical) comes out to be a future and promising tool for detecting early-stage caries and lesions on the surface of the teeth, where microleakage occurs at the interface of tooth restoration. Lesions can be shown because of their larger photoacoustic signal amplitudes in comparison to those given by healthy enamel. Moreover, the profoundness of a lesion can be determined from a time-reversal reconstruction imaging. This method has many advantages compared to traditional ultrasound such as better contrast between the caries or lesion and healthy tooth structure and no need for a coupling medium between the source and the tooth.

\section{REFERENCES}

1. Namitha J, Shashi Kiran M, Pallavi Nanaiah K, Naveen Jayapal, Vidya S, Gaurav Shetty. Photoacoustic imaging: an orchestra of light and sound. Journal of Evolution of Medical and Dental Sciences. 2013;45:8713-8723.

2. Yao J, Wang LV. Photoacoustic tomography: fundamentals, advances and prospects. Contrast Media Mol Imaging. 2011;6:332-345

3. Mehrmohammadi M, Yoon SJ, Yeager D, Emelianov SY. Photoacoustic Imaging for Cancer Detection and Staging. Curr Mol Imaging. 2013;2:89105.

4. Guo Z, Li L, Wang LV. On the speckle-free nature of photoacoustic tomography. Med Phys. 2009;36:4084-4088.

5. Wang LV. Multiscale photoacoustic microscopy and computed tomography. Nat Photonics. 2009;3:503-509.

6. Maslov K, Zhang HF, Hu S, Wang LV. Optical-resolution photoacoustic microscopy for in vivo imaging of single capillaries. Opt Lett. 2008;33:929931.

7. Bin R, Xin C, Favazza C, et al. Photoacoustic microscopy of human teeth Lasers in Dentistry XVII. [Proceedings Article].

8. Cheng R, Shao J, Gao X, Tao C, Ge J, Liu X. Noninvasive Assessment of Early Dental Lesion Using a Dual-Contrast Photoacoustic Tomography. Sci Rep. 2016;6:21798.

9. Yamada A, Kakino S, Matsuura Y. Detection of Photoacoustic Signals from Blood in Dental Pulp. Optics and Photonics Journal. 2016;6:229-236.

10. Hughes DA, Sampathkumar A, Longbottom C, Kirk KJ. Imaging and detection of early stage dental caries with an all-optical photoacoustic microscope. Journal of Physics: Conference Series. 2015;581:012002.

11. Hughes DA, Girkin JM, Poland S, et al. Investigation of dental samples using a $35 \mathrm{MHz}$ focussed ultrasound piezocomposite transducer. Ultrasonics. 2009;49:212-218.

12. Wang LV. Prospects of photoacoustic tomography. Med Phys. 2008;35:5758-5767. 